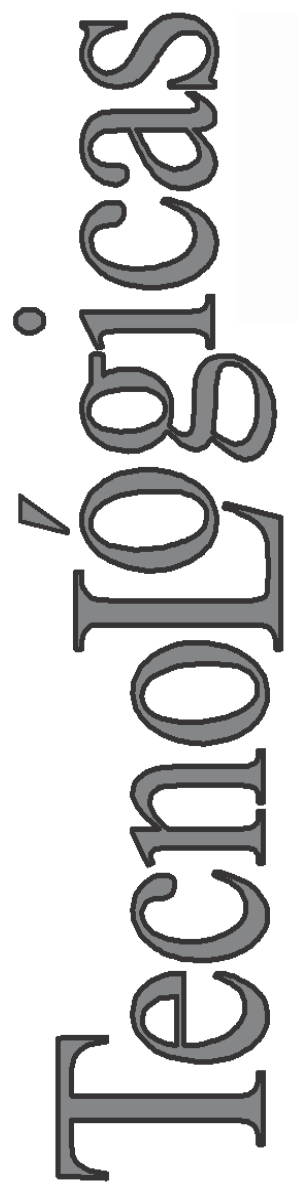

\title{
Diseño de una Estación de Trabajo para Personas con Discapacidad en Miembros Superiores Usando una Interfaz Cerebro Computador
}

\section{Design of a Workstation for People with Upper-Limb Disabilities Using a Brain Computer Interface}

John E. Muñoz-Cardona ${ }^{1,3}$ Cristian D. Muñoz-Cardona ${ }^{1,2}$ Oscar A. Henao- Gallo ${ }^{1,3}$

1 HCI Group, Unidad de Acción Motora, Clínica de Dolor del Eje Cafetero, Pereira-Colombia jemunozc@misena.edu.co

2 Programa de Ingeniería Industrial, Facultad de Ingeniería Industrial, Universidad Tecnológica de Pereira, Pereira-Colombia cdmunoz@utp.edu.co

3 Grupo de Investigación y Desarrollo en Cultura de la Salud, Facultad de ciencias de la Salud, Universidad Tecnológica de Pereira, Pereira-Colombia oscarhe@utp.edu.co 


\title{
Resumen
}

El presente trabajo presenta el diseño de una estación de trabajo para la inclusión laboral de personas en estado de discapacidad en miembros superiores. El sistema involucra el uso de una novedosa interfaz cerebro computador que sirve como puente entre el usuario y el ordenador. Nuestro objetivo es dilucidar los aspectos funciona- les, tecnológicos, ergonómicos y procedimentales de la puesta en marcha de la estación de trabajo; con el fin de romper con las barreas que imposibilitan el acceso a las herramientas TIC's y el trabajo por parte de las personas en estado de discapacidad en miembros superiores. Se encontró que la facilidad de acceso, la ergonomía, la adaptabilidad y la portabilidad de la estación de trabajo son los criterios de diseño más importantes. La implementación de este prototipo en una ambiente laboral tiene una TIR estimada de $43 \%$ para retribución. Finalmente se describe una tipología de servicios que podrían ser los más indicados para el proceso de inclusión laboral: telemarketing, televentas, encuestas telefónicas, toma de pedidos, ayuda social en catástrofes, información general y consultas, reservaciones en sitios turísticos, soporte técnico, emergencias, asistencia en línea y servicios posventa.

\section{Palabras clave}

Discapacidad; interfaz cerebro computador; diseño; estación de trabajo.

\begin{abstract}
This paper shows the design of work-station for work-related inclusion people upper-limb disability. The system involves the use of novel brain computer interface used to bridge the user-computer interaction. Our hope objective is elucidating functional, technological, ergonomic and procedural aspects to runaway operation station; with propose to scratch barrier to impossibility access to TIC's tools and work done for individual disability person. We found access facility ergonomics, adaptability and portable issue of workstation are most important design criteria. Prototype implementations in workplace environment have TIR estimate of $43 \%$ for retrieve. Finally we list a typology of services that could be the most appropriate for the process of labor including: telemarketing, telesales, telephone surveys, order taking, social assistance in disasters, general information and inquiries, reservations at tourist sites, technical support, emergency, online support and after-sales services.
\end{abstract}

\section{Keywords}

Disabled; brain-computer interface; design; workstation. 


\section{INTRODUCCIÓN}

Los jóvenes y adultos en situación de discapacidad del país viven una gran problemática debido a la falta de apoyo y oportunidades laborales evidencia- da mayormente en personas con discapacidad motriz en miembros superiores (Gómez, 2010); esta situación genera una clara discriminación y exclusión social lo que comúnmente conlleva a problemas de inconformidad mucho más profundos. Estas personas se encuentran en situación de alto grado de vulnerabilidad frente a la pobreza y violencia física, causado por la dificultad en el acceso a servicios de salud, educación y oportunidades laborales, al abuso emocional y al desconocimiento de su valor como individuos capaces y pensantes, sometiéndolos al aislamiento y la negación de su existencia, siendo esta última el problema ético y moral con mayor trasfondo que viven las personas discapacitadas y que se evidencia día tras día (Samaniego, 2006).

En Colombia se estima que un $16 \%$ de la población tiene alguna discapacidad y un 6\% discapacidad severa (personas con más del $50 \%$ de pérdida de su capacidad funcional). El $80 \%$ de la población con discapacidad severa no recibe atención suficiente del estado y no logra una real y efectiva integración social. No existen programas que cubran integralmente sus necesidades y solo los menores de 18 años tienen alguna mínima oportunidad de integración escolar o de formación profesional. Hay un serio deterioro familiar resultado de la discapacidad de alguno de sus miembros pues por cada persona en situación de discapacidad severa dos o más de sus familiares deben sacrificar su trabajo algunos casos la dedicación del cuidador, casi siempre madre en edad económicamente productiva, es de tiempo total. En el eje cafetero -objeto de intervención- no existen entidades que cubran cabalmente esta necesidad. Solo hay acciones aisladas de personas o instituciones de orientación asistencialista y desde el gobierno se generan acciones puntuales que no apoyan la organización comunitaria sino que resuelven algunos problemas individuales.

Por su parte, con el fin de crear un medio de comunicación más efectivo entre el hombre y la máquina, y lograr un mejor uso de las capacidades computacionales de los equipos vanguar- 
distas, las interfaces cerebro-computador (BCI: Brain Computer Interfaces) han sido desarrolladas en diferentes partes del mundo siempre buscando aprovechar al máximo las capacidades y habilidades intelectuales humanas (Tan \& Nijholt, 2010). Estas interfaces abren un mundo de posibilidades para la creación de tecnologías asistivas que les permitan a personas en estado de discapacidad, acceder a todos los beneficios y prestaciones que puede presentar un ordenador en línea (Carlson \& Millán, 2013). Este trabajo describe el proceso de diseño de una estación de trabajo creada para discapacitados en miembros superiores, la cual pretende convertirse en una herramienta de inclusión laboral que pueda ser envuelta en ambientes en donde se requiera del uso de un ordenador para tareas como dar clics, escribir números, navegar entre ventanas, etc.

\subsection{Interfaces Cerebro Computador}

Una interfaz cerebro computador (BCI) ofrece una alternativa de comunicación y control natural entre el cerebro y dispositivos externos, se define como la ciencia y la tecnología de dispositivos y sistemas de respuesta a los procesos neuronales en el cerebro que generan movimientos motores y procesos cognitivos. En lugar de depender de señales nerviosas o musculares, una BCI mide directamente la actividad cerebral asociada con la intención del usuario y traduce esta actividad grabada en señales correspondientes de control para diferentes aplicaciones (Graimann et al., 2010).

El casco Emotiv Epoc es una BCI que tiene incorporado 14 extensiones de electrodos (7 pares), la mayoría centrados alrededor de la parte frontal de la cabeza, contiene un acelerómetro de 2 ejes, un sistema de transmisión WiFi con su programación propia, lo que lo convierte en un dispositivo inalámbrico y una herramientas para la interacción a través de atención selectiva; inicialmente fue vendido como un dispositivo de juego para el sistema operativo Windows (Lang, 2012), sin embargo debido a su portabilidad y bajo costo ha sido usado para múltiples usos desde su salida al mercado en el año 2008. El neurocasco ha sido utilizado como herramienta para el manejo de aplicaciones de escritorio 
con interfaces de usuario especialmente diseñadas para que personas en estado de discapacidad puedan navegarlas y controlarlas a través de un pequeño número de acciones (particularmente gestos faciales) (Rami, 2012). Desde la Clínica de Dolor del Eje Cafetero en Pereira, se realizan investigaciones basadas en el uso del neurocasco Emotiv EPOC para la neurorehabilitación de pacientes con patologías como el accidente cerebro vascular y el ADHD (Muñoz et al., 2013).

\subsection{Marco Legal de Discapacidad}

Entre algunos de los artículos y leyes para la inclusión de personas en estado de discapacidad se encuentran:

La ley 361 de 1997, en la cual se crearon mecanismos para integrar a las personas con alguna limitación, entre ellas una serie de estímulos tributarios y ventajas competitivas para quien les vincule mediante contrato de trabajo: deducción en la renta, menor cuota de aprendices, ventajas para la empresa en licitaciones públicas, créditos con entidades estatales, preferencia de sus productos adquiridos por el estado.

La ley estatutaria 1618 del 2013, la cual dicta lineamientos a el Ministerio de Trabajo, a el Servicio Nacional de Aprendizaje SENA y a el Ministerio de Tecnologías de la Información y las Comunicaciones (TIC) acerca de las normatividades y estrategias encaminadas a ayudar a las personas con discapacidad, en términos de igualdad de oportunidades, equidad e inclusión en todos los campos. El Ministerio de Trabajo deberá: garantizar la capacitación y formación al trabajo de las personas con discapacidad y sus familias, fortalecer el programa de ubicación laboral de las personas con discapacidad, desarrollar planes y programas de inclusión laboral, fomentar la creación y fortalecimiento de unidades productivas, incentivar el desarrollo de negocios inclusivos y fortalecer el emprendimiento y crecimiento empresarial de las entidades que pro- penden por la independencia y superación de la población con discapacidad entre otros. El Servicio Nacional de Aprendizaje, SENA, deberá: garantizar asesoría y acompañamiento a los empresarios que deseen contratar personas con discapacidad, asegurar la capacitación y formación al trabajo de 
las personas con discapacidad, Fortalecer el Servicio Nacional de Empleo SNE de cada Regional para que garantice el acceso y beneficio de las personas con discapacidad, otorgar títulos de formación profesional en diferentes áreas a partir del reconocimiento de los procesos formativos que realizan las organizaciones de y para personas con discapacidad. Ministerio de Tecnologías de la Información y las Comunicaciones deberá: Dar estricto cumplimiento a las normas vigentes sobre accesibilidad y acceso a la información en los medios de comunicación, desarrollar programas que faciliten el acceso a las Tecnologías de la Información y las Comunicaciones, promover estrategias diversas, comunicación y educación permanentes, diseñar formas de divulgación accesibles para personas con discapacidad (Ley Estatutaria No. 1618, 2013).

\section{METODOLOGíA}

Se propone aportar metodologías y técnicas con base en la integración y reintegración de personal con discapacidad de las extremidades superiores a un ambiente laboral en donde realicen actividades en un equipo de cómputo.

Se desea atacar el problema de exclusión laboral de personas con discapacidad en las extremidades superiores, generando una plataforma integrada de alta tecnología que facilite la interacción de este tipo de usuarios con equipos y con la interfaz cerebro computador Emotiv Epoc. Los aspectos funcionales, tecnológicos y ergonómicos de la estación de trabajo se enmarcan dentro de la tarea más significativa que debe cumplir: romper con las barreas que imposibilitan el acceso a las herramientas TIC's por parte de las personas en estado de discapacidad en miembros superiores. Esta ruptura ocasiona un efecto domino sobre el entorno y la percepción de la industria sobre este tipo de personas, transformando todo estigma, aislamiento y exclusión, en una nueva oportunidad de negocio.

Se acudió a la red Tecnoparque del SENA, para el diseño inicial de la estación de trabajo. Desde allí, se lograron establecer algunos de los criterios para el diseño de la estación de trabajo 
entre los cuales se destaca, la facilidad de acceso (relacionada a los materiales de la superficie de trabajo), la ergonomía, la adaptabilidad y la portabilidad de toda la estación de trabajo. Se realizó un "benchmarking" sobre estaciones de trabajo de tipo modular que pueden ser adaptadas para personas discapacitadas, encontrándose que la mayoría de estos puestos de trabajo no poseen las condiciones mínimas de conformidad para personas en estado de discapacidad y que podrían llegar a imposibilitar la maniobrabilidad de dispositivos propios de la estación de trabajo propuesta. Sin embargo existe una denominada Nethrone, la cual brinda una posición de descanso, a fin de aliviar el estrés de la espalda. La estructura ovalada cuenta con cinco actuadores eléctricos para ajustar el asiento reclinable y es la que más se ajusta a los requerimientos de diseño planteados.

Se escogió el sensor Emotiv Epoc porque es verdaderamente económico comparado con un sistema EEG médico y a diferencia de la utilización de los cables en los exámenes de EEG, el neurocasco es totalmente inalámbrico, permitiendo así al usuario moverse libre y naturalmente. Tiene un tiempo de autonomía cercano a las 8 horas, el sistema BCI también incluye un acelerómetro de dos ejes el cual permite el mapeo del movimiento de la cabeza en las coordenadas del mouse de la pantalla. Existen varios tipos de señales que pueden ser capturadas mediante el neurocasco con las cuales el usuario puede generar una interacción efectiva con aplicaciones de escritorio: (1) gestos faciales capturados mediante los electrodos AF3, AF4, F3, F4, F7 y F8, ubicados en el lóbulo frontal del cerebro, los cuales producen señales electromiográficas que pueden ser caracterizadas con mayor facilidad, (2) ritmos sensoriomotores, los cuales son oscilaciones registradas en bandas de frecuencia específicas que son dinámicas y cambian con respecto a diferencias de estados de ánimo del usuario, estados de concentración o relajación, imaginación de movimiento, entre otras (Badcock et al., 2013), (3) potenciales evocados, los cuales son cambios de la señal generalmente provocados por estímulos visuales detectados en los electrodos del lóbulo occipital, en cuyo caso son llamados potenciales evocados visuales; estos potenciales pueden ser caracteri- 
zados y clasificados (mediante algoritmos) para cada usuario en respuesta a estímulos visuales proyectados sobre una pantalla.

Se realizó un análisis de costos para la implementación de este tipo de estaciones de trabajo, arrojando una tasa interna de retorno (TIR) de la inversión para el primer año cercana al 43\%, cifra que fortalece enormemente la idea y sirve como criterio de para el desarrollo de la propuesta económica final.

\section{RESULTADOS Y DISCUSIÓN}

El desarrollo completo de la estación de trabajo puede ser dividido en dos partes funcionales: el hardware o elemento físico $\mathrm{y}$ el software o elemento no tangible. En la parte del hardware se estableció de forma preliminar un esquema funcional de la estación de trabajo, lo que permitió identificar cada uno de los elementos que son requeridos para la estación de trabajo (ver Fig. 1).

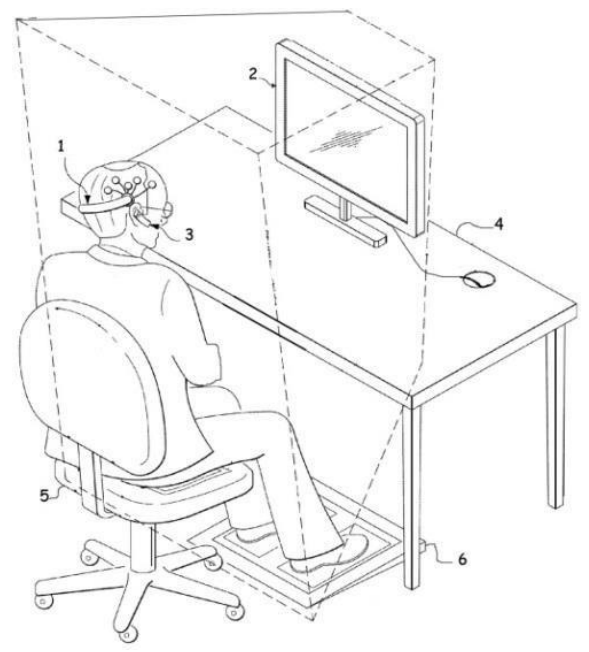

Fig. 1. Esquema funcional de la estación de trabajo propuesta

(1) Sistema BCI no invasivo, totalmente inalámbrico, permitiendo así al usuario moverse libre y naturalmente. 
(2) Computador todo en uno, el cual permite la combinación de la CPU y monitor en un solo diseño, eliminando todos los cables que los unen y optimizando los espacios.

(3) Auriculares Inalámbricos con micrófono: auriculares con cinta trasera y plegable, fácil de guardar, diseño liviano y ergonómico, perfecto para largos periodos de uso. Cuenta con un micrófono de alta sensibilidad y una muy buena respuesta de frecuencia.

(4) Superficie de trabajo: puesto ergonómico y adaptado para el personal discapacitado.

(5) Asiento ajustable: asiento ergonómico ajustable a las necesidades del personal discapacitado.

(6) Pedales: pedales de accionar inferior, útiles para ajustar el ángulo de inclinación de la silla y/o otras funciones para mejorar el confort.

Adicionalmente, se desarrolló un software en versión beta de la aplicación para la integración del sistema BCI con el ordenador. Este software fue probado en personas sanas y se logró establecer un protocolo para su implementación, basado en tres etapas las cuales son resumidas a continuación:

Etapa de configuración: se requiere que previamente el sistema BCI esté cargado, que sus almohadillas se encuentren en perfecto estado y sus electrodos se encuentren adheridos con el cuero cabelludo, antes de conectar se recomienda hidratar las almohadillas con al menos 2 gotas de solución salina por cada una. Se realiza la instalación del software en el ordenador y se verifica la calidad de la conexión de los electrodos según el código de colores propuesto por el fabricante (de mayor a menor conductividad: Verde, Amarillo, Rojo, Negro).

Etapa de entrenamiento: una vez el usuario tenga conectado el sistema BCI y el ordenador esté configurado correctamente, se iniciará el proceso de entrenamiento, el cual consiste en usar la Expressive Suite del neurocasco con el fin de asignar un gesto facial particular al evento del clic necesario para operar el software específico. Al usuario entonces se le pide realizar una a una las siguientes expresiones faciales: sonreír, guiñar el ojo derecho, guiñar el ojo izquierdo, mirar hacia la derecha, mirar hacia la 
izquierda, parpadear, apretar los dientes o sonreír. El sistema registra la actividad bioeléctrica producida por cada una de las expresiones faciales; el evento del clic del mouse debe ser asignado a la expresión que más domine el usuario para generar la interacción.

Etapa de implementación: una vez configurado correctamente el sensor y habiendo entrenado al usuario, se procede a realizar el proceso de navegación con el ordenador. Mediante el acelerómetro del sensor, se mapean los movimientos de la cabeza del usuario y se le asignan al movimiento $2 \mathrm{D}$ del cursor en la pantalla, el usuario dirige el cursor hasta donde quiere realizar el clic y realiza la expresión entrenada. Finalmente para opciones de teclado, se utiliza un teclado virtual gratuito para rellenar campos con letras y números o se utiliza el micrófono para realizar búsquedas en el navegador de Google Chrome a través de las herramientas de reconocimiento de voz.

En un último estado se realizó un análisis de requerimientos de software y aplicabilidad de la estación de trabajo propuesta, la cual arrojó una tipología de servicios que podrían ser los más indicados para el proceso de inclusión laboral, como son: telemarketing, televentas, encuestas telefónicas, toma de pedidos, ayuda social en catástrofes, información general y consultas, reservaciones en sitios turísticos, soporte técnico, emergencias y asistencia en línea y servicios posventa.

\section{CONCLUSIONES}

La implementación de una interfaz cerebro computador como herramienta para la inclusión laboral de personas con discapacidad en miembros superiores, se presenta como una opción rentable para aprovecharlos beneficios tributarios ofrecidos por el gobierno. El tipo de servicios que mejor podrían desempeñar los usuarios de esta estación de trabajo serían: telemarketing, encuestas telefónicas, toma de pedidos, ayuda social en catástrofes, información general y consultas, reservaciones en sitios turísticos, soporte técnico, emergencias y asistencia en línea y servicios posventa. 
La estación de trabajo diseñada propone una TIR de 43\% para el primer año cifra que fortalece enormemente la idea y sirvió como criterio de para el desarrollo de la propuesta económica final.

\section{AGRADECIMIENTOS}

Este trabajo ha sido posible gracias al trabajo académico realizado por el semillero de Investigación en HCI y la Clínica de Dolor del Eje Cafetero.

\section{REFERENCIAS}

Badcock N., Mousikou P., Mahajan Y., Lissa P., Thie J., McArthut G., (2013). Validation of the Emotiv EPOC EEG gaming system for measuring research quality auditory ERPs. PeerJ 1:e38; DOR 107717/peerj.38.

Carlson, T., Millán J. R., (2013). Brain-controlled wheelchairs: a robotic architecture. IEEE Robot and Autom Magaz, 20(1): 65-73, 2013.

Gómez, J.C. (2010). Discapacidad en Colombia: Reto para la Inclusión en Capital Humano, Fundación Saldarriaga Concha, Bogotá 2010.

Graimann, B., Allison B., Pfurtshceller G., (2010). Brain-Computer Interfaces, Revolutionizing Human-Computer Interaction. The Front Colle Springer, pp.151, 2010.

Lang M.,(2012). Investigating the Emotiv Epoc for cognitive control in limited training time.

Dep of Comp Sci, Univ of Canterb, 2012.

Ley estatutaria No. 1618, "Por medio de la cual se establecen las disposiciones para garantizar el pleno ejercicio de los derechos de las personas con discapacidad", disponible en la web, revisión (20 de Septiembre de 2013): http://wsp.presidencia.gov.co/Normativa/Leyes/Documents/2013/LEY \%201618\%20DEL\%2027\%20DE\%20FEBRERO\%20DE\%202013.pdf

Muñoz J., Lopez J.F., Henao O., J.F. Villada., (2013). BKI: Brain Kinect Interface, a new hybrid BCI for rehabilitation. 3rd Conference in Games For Health Europe, Amsterdam 2013.

Rami P., (2012). Apparatus for operating a computer using thoughts or facial impressions. Eur Patent App, EP 2447808 A1, 2012. 
Samaniego P., (2006). Aproximación a la realidad de las personas con discapacidad en Latinoamerica. Com Españ de Repres de Pers con Disc., Madrid 2006.

Tan, D.S., Nijholt A., (2010). Brain-Computer Interfaces, Applying our Minds to Human

Computer Interaction. Hum Comp Inter Series, pp. 100, 2010. 